\title{
Corneal ulcerative disease in dogs under primary veterinary care in England: epidemiology and clinical management
}

\author{
Dan G. O'Neill ${ }^{1 *}$, Monica M. Lee ${ }^{2}$, Dave C. Brodbelt ${ }^{1}$, David B. Church ${ }^{3}$ and Rick F. Sanchez ${ }^{4}$
}

\begin{abstract}
Background: Corneal ulcerative disease (CUD) has the potential to adversely affect animal welfare by interfering with vision and causing pain. The study aimed to investigate for the first time the prevalence, breed-based risk factors and clinical management of CUD in the general population of dogs under primary veterinary care in England.

Results: Of 104,233 dogs attending 110 clinics participating within the VetCompass Programme from January $1^{\text {st }}$ to December $31^{\text {st }} 2013$, there were 834 confirmed CUD cases (prevalence: $0.80 \%$, 95\% confidence interval (Cl) 0.75-0.86). Breeds with the highest prevalence included Pug (5.42\% of the breed affected), Boxer (4.98\%), Shih Tzu (3.45\%), Cavalier King Charles Spaniel (2.49\%) and Bulldog (2.41\%). Purebred dogs had 2.23 times the odds $(95 \% \mathrm{Cl} 1.84-2.87$, $P<0.001)$ of CUD compared with crossbreds. Brachycephalic types had 11.18 (95\% Cl 8.72-14.32, $P<0.001)$ and spaniel types had 3.13 (95\% Cl 2.38-4.12, $P<0.001$ ) times the odds for CUD compared with crossbreds. Pain was recorded in 385 (46.2\%) cases and analgesia was used in 455 (54.6\%) of dogs. Overall, 62 (7.4\%) cases were referred for advanced management and CUD contributed to the euthanasia decision for 10 dogs.
\end{abstract}

Conclusions: Breeds such as the Pug and Boxer, and conformational types such as brachycephalic and spaniels, demonstrated predisposition to CUD in the general canine population. These results suggest that breeding focus on periocular conformation in predisposed breeds should be considered in order to reduce corneal disease.

Keywords: General practice, First opinion, VetCompass, Ulcerative keratitis, Corneal ulceration, Brachycephalic, Spaniel

\section{Plain English summary}

The cornea is the outermost layer of the eye and it must remain transparent to support vision. Ulcerated corneas can lose transparency and become particularly painful. Understanding which breeds and conformational types of dog in primary-care practices are commonly affected by corneal disease could assist in developing preventive measures such as breeding strategies or improved eye care and guide veterinary surgeons in primary-care practice. Information collected directly from the clinical records of first opinion veterinary practices by the VetCompass Programme can provide reliable health data on

\footnotetext{
* Correspondence: doneill@rvc.ac.uk

'Pathobiology and Population Sciences, The Royal Veterinary College,

Hawkshead Lane, North Mymms, Hatfield, Herts AL9 7TA, UK

Full list of author information is available at the end of the article
}

the general population of dogs in the UK, and was used here for the first time to explore the clinical picture associated with 'corneal ulcerative disease.

Of 104,233 dogs attending 110 primary-care practices in England, there were 834 dogs with corneal ulceration $(0.80 \%$ overall). The breeds with the highest prevalence were the Pug $(5.42 \%$ of the breed affected), Boxer (4.98\%), Shih Tzu (3.45\%), Cavalier King Charles Spaniel (2.49\%) and Bulldog (2.41\%). Pain associated with the disease was recorded in $46.2 \%$ of cases, at least one painkiller was dispensed or administered in 54.6, and 17.0\% underwent surgery.

Compared with crossbred dogs, the Pug was 19 times as likely and the Boxer was 12 times as likely to have corneal ulceration. In comparison with crossbred dogs, flat-faced dogs (brachycephalics) were 11 times as likely and spaniel types were 3 times as likely to have corneal ulceration. 
These results demonstrate for the first time by using large numbers of primary-care practice cases that brachycephalic breeds have a predisposition for corneal ulcerative disease. The strong breed predispositions suggests the opportunity to improve animal welfare by focusing on breed-based preventive strategies.

\section{Background}

The outermost layer of the cornea is a richly innervated epithelium that protects the underlying stroma and supports the endothelium in maintaining a dehydrated stroma, which is essential for corneal transparency [1]. Epithelial defects that affect the basement membrane and expose the corneal stroma are known as corneal ulcers [2]. Corneal ulcerative disease (CUD) can affect animal welfare by the development of pain, reflex uveitis, perforation and even loss of the eye and possibly also by temporarily or permanently interfering with vision $[3,4]$.

The majority of published epidemiological studies on canine CUD are based on referral populations and therefore are biased towards selection of more complicated cases and towards outcomes that are heavily influenced by the post-referral skill-sets and equipment [5-10]. Although findings from referral studies may be useful for referral practitioners, these results are likely to be poorly generalisable to general primary-care caseloads and have limited applicability for quantifying disorder levels in broader dog populations [11]. It is therefore important for ophthalmologists, general clinicians and welfare scientists to access clinical research results from primarycare practice in order to offer informed advice relevant to the general primary-care CUD caseloads even if, and especially where, the standards of care differ from the norms in referral practice [12]. Welfare scientists can benefit from access to primary-care prevalence data that can assist with disorder prioritisation across all dogs and from access to breed risk factor data that can assist with focused prioritisation within individual breeds [12-14].

Corneal ulcerative disease describes a broad clinical presentation resulting from a variety of underlying causes and predispositions that may or may not be formally identified during the clinical work-up [15]. Primary causes of CUD in dogs include spontaneous chronic corneal ulceration (SCCED) $[5,10,16]$ and canine herpes virus-1 [17]. Multiple secondary causes are reported, including entropion [18, 19], ectopic cilia $[8,20]$, primary and secondary forms of keratoconjunctivitis sicca (KCS) $[7,21]$, corneal degeneration [22], traumatic events [23-25], corneal overexposure related to general anesthesia [26], facial nerve paralysis [27] and orbital diseases $[28,29]$. Many of these factors have also been associated with certain breed phenotypes [30] and some smaller studies based on referral populations of less than 250 cases have reported increased CUD prevalence in breed-types such as brachycephalic [7, 31, 32] or spaniel types [7, 33]. However, CUD has not been conclusively associated with particular breed-types or conformational signalment in the general dog population mainly because general population studies with large enough numbers of cases have been lacking to date. The present study aimed to investigate the prevalence, risk factors and clinical management for CUD as diagnosed by veterinary practitioners in the general population of dogs attending primary-care practices enrolled in the UK VetCompass Programme in order to give a picture of the occurrence, diagnostics and outcomes of the condition in this setting. It was hypothesized that brachycephalic and spaniel types have higher odds of CUD than crossbred dogs.

\section{Methods}

The VetCompass Programme collates de-identified electronic patient record (EPR) data from primary-care veterinary practices in the UK for epidemiological research [34]. Collaborating practices can record summary diagnosis terms from an embedded VeNom Code [35] list during episodes of care. VetCompass collects information fields that include species, breed, date of birth, sex, neuter status, insurance status and bodyweight, and clinical information from free-form text clinical notes and summary diagnosis terms (VeNom codes), plus treatment and deceased status with relevant dates. The EPR data were extracted from practice management systems using integrated clinical queries and uploaded to a secure VetCompass structured query language database [36].

A cross-sectional analysis using cohort clinical data of dogs attending VetCompass practices was used to estimate the prevalence, risk factors and clinical management for CUD [37]. The sampling frame for the current study included dogs under veterinary care within the VetCompass database from January $1^{\text {st }} 2013$ to December $31^{\text {st }}$ 2013. Dogs 'under veterinary care' were defined as any dog that had either at least one EPR recorded from January $1^{\text {st }}$ to December $31^{\text {st }} 2013$ or, alternatively, at least one EPR both before and after 2013. Sample size calculations estimated that 2,958 dogs of a specific type (e.g., brachycephalic or spaniel) and 11,832 crossbred dogs would be required to detect an odds ratio of 2.0 times or greater for CUD assuming a $0.5 \%$ prevalence of CUD in the crossbred dogs (4:1 ratio of crossbred:specific type, two-sided $95 \%$ confidence interval, $80 \%$ power) [38]. Ethical approval was granted by the RVC Ethics and Welfare Committee (reference number 2015/T94).

Case inclusion criteria required that a final diagnosis of CUD was recorded in the EPR and that corneal ulceration was present during the 2013 study period. Case-finding involved initial screening of all EPRs for candidate CUD cases by searching the clinical free-text field using search terms including fluo and pos, fluo and +, corn and ulc, 
eyelid flap, TEF, keratect, descem, and the VeNom term field using the search term corneal ulcer. The candidate cases were randomly ordered by the Microsoft Excel RAND function (Microsoft Office Excel 2007, Microsoft Corp.) and the clinical notes of each candidate were manually reviewed in detail to evaluate them for case inclusion. All dogs with confirmed CUD were grouped as CUD cases for the analysis, and all remaining study dogs were grouped as non-cases. Additional data were extracted on confirmed CUD cases including the date of first diagnosis, history of previous CUD events, laterality of eye/s affected, diagnostic tests used, whether pain was recorded in the clinical notes or not, types of medical and surgical therapy used, whether the animal was referred for advanced case management, CUD clinical status at the final record available, CUD recurrence in the same eye and whether CUD was associated with decisions to euthanase.

A purebred variable categorised all dogs of recognisable breeds as 'purebred' and the remaining dogs as 'crossbred' [39]. A breed variable included individual breeds with 10 or more CUD cases, a grouped category of all remaining purebreds and a general grouping of crossbred dogs. This approach was taken to allow focus on commonly affected breeds and to facilitate statistical power for the individual breed analyses [40]. A spaniel-brachycephalic variable grouped dogs into four categories: spaniel, brachycephalic, other purebreds or crossbred. Spaniel breeds were selected based on the inclusion of the word 'spaniel' in their name and included American Cocker, Brittany, Cavalier King Charles, Clumber, English Cocker, English Springer, English Toy, Field, Japanese (also known as Japanese Chin), King Charles, Picardy, Sussex, Tibetan, Welsh Cocker, Working Cocker, Water Spaniel and Welsh Springer. Brachycephalic breeds were selected based on breeds commonly included in studies on brachycephaly [41-44] and included American Bulldog, Boston Terrier, Boxer, English Bulldog, French Bulldog, Pekingese, Pug, Shih Tzu and Victorian Bulldog. A Kennel Club breed group variable classified breeds recognised by the UK Kennel Club into their relevant breed groups (gundog, hound, pastoral, terrier, toy, utility and working) and all remaining types were classified as non-Kennel Club recognised [45]. $A$ neuter variable described the status of the $\operatorname{dog}$ (neutered or entire) recorded at the final EPR. An insurance variable described whether a dog was insured at any point during the study period. An age variable categorised age (years) into six groups $(<3.0,3.0-5.9,6.0-8.9,9.0-11.9, \geq 12.0$, not recorded). Age (years) was calculated for case animals at the date of diagnosis of the current corneal ulceration event and for non-case dogs at either July $1^{\text {st }}, 2013$ for dogs born before this date or at December $31^{\text {st }}, 2013$ otherwise. An adult bodyweight variable categorised adult bodyweight into six groups $(0.0-9.9 \mathrm{~kg}, 10.0-19.9 \mathrm{~kg}$, $20.0-29.9 \mathrm{~kg}, 30.0-39.9 \mathrm{~kg}, \geq 40.0 \mathrm{~kg}$, not available). Adult bodyweight described the maximum bodyweight recorded during the study period for dogs older than 9 months. A bodyweight relative to breed mean variable characterised the adult bodyweight of individual dogs as either below or equal/above the mean adult bodyweight for their breed and sex within the overall study population. This variable allowed the effect of adult bodyweight to be assessed within each breed/sex combination.

Following data checking and cleaning in Excel (Microsoft Office Excel 2013, Microsoft Corp.), analyses were conducted using Stata Version 13 (Stata Corporation). The 1year period prevalence with 95\% confidence intervals (CI) described the probability of CUD at any time during the 1year 2013 study period. The CI estimates were derived from standard errors, based on approximation to the normal distribution [46]. Descriptive statistics characterised the purebred status, breed, Kennel Club breed group, spanielbrachycephalic, sex, neuter status, insurance, age, adult bodyweight and bodyweight relative to breed mean for the case and non-case dogs. Clinical management regimes were reported and analysed for the CUD cases using the chisquare test to compare categorical variables and the MannWhitney $U$ test to compare continuous variables [46].

Binary logistic regression modelling was used to evaluate univariable associations between risk factors (purebred, breed, Kennel Club breed group, spaniel-brachycephalic, adult bodyweight, bodyweight relative to breed mean, age, sex, neuter and insurance) and diagnosis of CUD. Because breed was a factor of primary interest for the study, purebred, spaniel-brachycephalic and Kennel Club breed group (variables that are highly collinear with breed) and adult bodyweight (a defining characteristic of individual breeds) were excluded from the initial breed multivariable modelling. Instead, each of these variables individually replaced the breed variable in the main final model in order to evaluate their effects after taking account of the other variables. Risk factors with liberal associations in univariable modelling $(P<0.2)$ were taken forward for multivariable evaluation. Model development used manual backwards stepwise elimination. Pair-wise interaction effects were evaluated for the final model variables and confounding effects from dropped variables were assessed by individual reintroduction to the final model. The area under the ROC curve was used to evaluate the quality of the model fit (non-random effect model) [47]. Statistical significance was set at $P<0.05$.

\section{Results}

The study population comprised 104,233 dogs attending 110 primary-care practices in England. Following manual review, 834 dogs met the CUD inclusion criteria giving an overall 1-year period prevalence of $0.80 \%$ (95\% CI 0.75-0.86). 
Individual breeds with the highest CUD prevalence included Pug (5.42\% of the breed affected, 95\% CI 4.117.00), Boxer (4.98\%, 95\% CI 3.89-6.26), Shih Tzu (3.45\%, 95\% CI 2.70-4.33), Cavalier King Charles Spaniel (2.49\%, 95\% CI 1.89-3.20) and Bulldog (2.41\%, 95\% CI 1.463.74) (Table 1). The CUD prevalence in the brachycephalic group was $3.76 \%(95 \%$ CI $3.30-4.25)$ and in the spaniel group was 1.31\% (95\% CI 1.10-1.56).

Of the CUD cases with complete data available for that variable, $746(89.5 \%)$ were purebred, $382(45.8 \%)$ were female, 436 (78.0\%) were neutered and 331 (63.5\%) were insured. Dogs with CUD had a median adult bodyweight of $12.7 \mathrm{~kg}$ (IQR: 8.6-25.0, range: 1.6-96.5) and median age at diagnosis overall of 7.20 years (IQR: 3.50 9.80 range: $0.0-19.0)$. The most common breeds among the CUD cases were Shih Tzu (70/834 cases, $8.4 \%$ of all cases), Boxer (69/834, 8.3\%), Staffordshire Bull Terrier (66/834, 7.9\%), Cavalier King Charles Spaniel (58/834, $7.0 \%)$ and Pug $(55 / 834,6.6 \%)$ along with crossbred dogs $(88 / 834,10.6 \%)$ (Table 2). Of the non-case dogs with complete data on the variable, $80,109(77.5 \%)$ were purebred, 49,180 (47.7\%) were female, 46,050 (76.9\%) were neutered and 31,840 (52.3\%) were insured. The median adult bodyweight for non-cases was 17.6 (IQR: 9.4-29.0, range: $1.1-109.0) \mathrm{kg}$ and the median age was 4.9 years
(IQR: 2.1-8.5, range: 0.0-31.2). The most common breeds among the non-case dogs were Labrador Retriever $(9,517,9.2 \%)$, Staffordshire Bull Terrier $(6,797$, 6.6\%), Jack Russell Terrier $(6,658,6.4 \%)$ and Cocker Spaniel $(4,197,4.1 \%)$ as well as $23,241(22.5 \%)$ crossbreds (Table 2). Data completeness varied between the variables assessed: breed $100.0 \%$, age $100.0 \%$, sex $99.7 \%$, bodyweight (aged $>9$ months) $81.8 \%$, insurance $58.9 \%$, and neuter $58.0 \%$.

Fluorescein staining was the most commonly used ancillary diagnostic aid, used in 774 (92.8\%) cases. Schirmer tear test-1 (STT-1) strips were used in 198 (23.7\%) cases, and bacteriology was used in 11 (1.3\%) cases. There were 53 (6.4\%) CUD cases diagnosed without the recorded use of any of these three tests. The laterality of the affected eye was recorded in 820 (98.3\%) of cases. The left eye was affected in 390 (46.8\%) cases, the right eye was affected in $382(45.8 \%)$ cases and both eyes were affected in $(5.8 \%)$ cases. Of the 834 confirmed CUD cases, $770(92.3 \%)$ had no recorded history of a prior CUD event. Of the 64 cases with a history of prior CUD, the same eye was previously affected in 29 $(45.5 \%)$, the contralateral eye was previously affected in $22(34.4 \%)$ and both eyes were previously affected in $13(20.3 \%)$.

Table 1 Prevalence of corneal ulcerative disease in commonly affected dog breeds

\begin{tabular}{|c|c|c|c|c|}
\hline Breed & No. cases & No. dogs in study & Prevalence \% & $95 \% \mathrm{Cl}^{\mathrm{a}}$ \\
\hline Pug & 55 & 1015 & 5.42 & $4.11-7.00$ \\
\hline Boxer & 69 & 1386 & 4.98 & $3.89-6.26$ \\
\hline Shih Tzu & 70 & 2031 & 3.45 & $2.70-4.33$ \\
\hline Cavalier King Charles Spaniel & 58 & 2332 & 2.49 & $1.89-3.20$ \\
\hline Bulldog & 19 & 787 & 2.41 & $1.46-3.74$ \\
\hline King Charles Spaniel & 11 & 496 & 2.22 & $1.11-3.93$ \\
\hline Lhasa Apso & 19 & 892 & 2.13 & $1.29-3.31$ \\
\hline French Bulldog & 12 & 642 & 1.87 & $0.97-3.24$ \\
\hline West Highland White Terrier & 28 & 2859 & 0.98 & $0.65-1.41$ \\
\hline Staffordshire Bull Terrier & 66 & 6863 & 0.96 & $0.74-1.22$ \\
\hline Cocker Spaniel & 37 & 4234 & 0.87 & $0.62-1.20$ \\
\hline English Springer Spaniel & 19 & 2210 & 0.86 & $0.52-1.34$ \\
\hline Border Terrier & 11 & 1327 & 0.83 & $0.41-1.48$ \\
\hline Chihuahua & 17 & 2223 & 0.76 & $0.45-1.22$ \\
\hline Yorkshire Terrier & 24 & 3354 & 0.72 & $0.46-1.06$ \\
\hline Jack Russel Terrier & 41 & 6699 & 0.61 & $0.44-0.83$ \\
\hline Border Collie & 12 & 2807 & 0.43 & $0.22-0.75$ \\
\hline Crossbreed & 88 & 23329 & 0.38 & $0.30-0.46$ \\
\hline Labrador Retriever & 24 & 9541 & 0.25 & $0.16-0.37$ \\
\hline Other purebreds & 154 & 29206 & 0.53 & $0.45-0.62$ \\
\hline Overall total & 834 & 104233 & 0.80 & $0.75-0.86$ \\
\hline
\end{tabular}

Prevalence of diagnosis of corneal ulcerative disease in commonly affected dog breeds attending primary-care veterinary practices in England ${ }^{\mathrm{a}} \mathrm{Cl}$ confidence interval 
Table 2 Descriptive and univariable logistic regression results

\begin{tabular}{|c|c|c|c|c|c|c|}
\hline Variable & Category & Case No. (\%) & Non-case No. (\%) & Odds ratio & $95 \% \mathrm{Cl}^{\mathrm{a}}$ & $P$-value \\
\hline \multirow[t]{2}{*}{ Purebred status } & Crossbred & $88(10.55)$ & $23243(22.49)$ & Base & & \\
\hline & Purebred & $746(89.45)$ & 80109 (77.51) & 2.46 & $1.97-3.07$ & $<0.001$ \\
\hline \multirow[t]{20}{*}{ Common breeds } & Crossbreed & $88(10.55)$ & $23241(22.48)$ & Base & & \\
\hline & Pug & $55(6.59)$ & $960(0.93)$ & 15.13 & $10.74-21.32$ & $<0.001$ \\
\hline & Boxer & $69(8.27)$ & $1317(1.27)$ & 13.84 & $10.05-19.06$ & $<0.001$ \\
\hline & Shih Tzu & $70(8.39)$ & $1961(1.90)$ & 9.43 & $6.86-12.95$ & $<0.001$ \\
\hline & Cavalier King Charles Spaniel & $58(6.95)$ & $2274(2.20)$ & 6.74 & $4.82-9.41$ & $<0.001$ \\
\hline & Bulldog & $19(2.28)$ & $768(0.74)$ & 6.53 & $3.96-10.78$ & $<0.001$ \\
\hline & King Charles Spaniel & $11(1.32)$ & $485(0.47)$ & 5.99 & $3.18-11.28$ & $<0.001$ \\
\hline & Lhasa Apso & $19(2.28)$ & $873(0.84)$ & 5.75 & $3.48-9.48$ & $<0.001$ \\
\hline & French Bulldog & $12(1.44)$ & $630(0.61)$ & 5.03 & $2.74-9.24$ & $<0.001$ \\
\hline & West Highland White Terrier & $28(3.36)$ & $2831(2.74)$ & 2.61 & $1.70-4.00$ & $<0.001$ \\
\hline & Staffordshire Bull Terrier & $66(7.91)$ & $6797(6.57)$ & 2.56 & $1.86-3.53$ & $<0.001$ \\
\hline & Cocker Spaniel & $37(4.44)$ & $4197(4.06)$ & 2.33 & $1.58-3.42$ & $<0.001$ \\
\hline & English Springer Spaniel & $19(2.28)$ & $2191(2.12)$ & 2.29 & $1.39-3.77$ & 0.001 \\
\hline & Border Terrier & $11(1.32)$ & $1316(1.27)$ & 2.21 & $1.18-4.14$ & 0.014 \\
\hline & Chihuahua & $17(2.04)$ & $2206(2.13)$ & 2.04 & $1.21-3.43$ & 0.008 \\
\hline & Yorkshire Terrier & $24(2.88)$ & $3330(3.22)$ & 1.90 & $1.21-2.99$ & 0.005 \\
\hline & Jack Russell Terrier & $41(4.92)$ & $6658(6.44)$ & 1.63 & $1.12-2.36$ & 0.010 \\
\hline & Border Collie & $12(1.44)$ & $2795(2.70)$ & 1.13 & $0.62-2.08$ & 0.684 \\
\hline & Labrador Retriever & $24(2.88)$ & $9517(9.20)$ & 0.67 & $0.42-1.05$ & 0.078 \\
\hline & Other purebreds & $154(18.47)$ & $29052(28.10)$ & 1.40 & $1.08-1.82$ & 0.012 \\
\hline \multirow[t]{8}{*}{ Kennel Club Breed Groups } & Breed not Kennel Club recognised & $139(16.67)$ & $31185(30.16)$ & Base & & \\
\hline & Toy & $188(22.54)$ & $12413(12.00)$ & 3.40 & $2.73-4.24$ & $<0.001$ \\
\hline & Utility & $151(18.11)$ & $8681(8.40)$ & 3.90 & $3.10-4.92$ & $<0.001$ \\
\hline & Terrier & $120(14.39)$ & $13537(13.09)$ & 1.99 & $1.56-2.54$ & $<0.001$ \\
\hline & Gundog & $101(12.11)$ & $20551(19.88)$ & 1.10 & $0.85-1.43$ & 0.456 \\
\hline & Hound & $22(2.64)$ & $4525(4.38)$ & 1.09 & $0.69-1.71$ & 0.706 \\
\hline & Pastoral & $31(3.72)$ & $7467(7.22)$ & 0.93 & $0.63-1.38$ & 0.721 \\
\hline & Working & $82(9.83)$ & $5040(4.87)$ & 3.65 & $2.77-4.80$ & $<0.001$ \\
\hline \multirow[t]{4}{*}{ Spaniel-brachycephalic } & Crossbred & $88(10.6)$ & $23243(22.5)$ & Base & & \\
\hline & Spaniel & $130(15.6)$ & $9887(9.6)$ & 3.47 & $2.65-4.56$ & $<0.001$ \\
\hline & Brachycephalic & $239(28.7)$ & $6124(5.9)$ & 10.31 & $8.06-13.18$ & $<0.001$ \\
\hline & Purebred other & $377(45.2)$ & $64098(62.0)$ & 1.55 & $1.23-1.96$ & $<0.001$ \\
\hline \multirow[t]{6}{*}{ Adult (> 9 months) bodyweight (kg) } & $<10.0$ & $264(31.65)$ & $24731(23.92)$ & 1.63 & $1.28-2.08$ & $<0.001$ \\
\hline & $10.0-19.9$ & $245(29.38)$ & $23047(22.29)$ & 1.59 & $1.25-2.03$ & $<0.001$ \\
\hline & $20.0-29.9$ & $145(17.39)$ & $18302(17.70)$ & 1.20 & $0.92-1.56$ & 0.186 \\
\hline & $30.0-39.9$ & $88(10.55)$ & $13301(12.86)$ & Base & & \\
\hline & $\geq 40.0$ & $32(3.84)$ & $6564(6.35)$ & 0.70 & $0.46-1.07$ & 0.097 \\
\hline & Not available & $60(7.19)$ & $17454(16.88)$ & 0.58 & $0.43-0.79$ & 0.001 \\
\hline \multirow[t]{4}{*}{ Bodyweight relative to breed mean } & Equal/Higher & $389(46.64)$ & $40713(39.37)$ & 1.12 & $0.97-1.29$ & 0.114 \\
\hline & Lower & $372(44.60)$ & $43689(42.25)$ & Base & & \\
\hline & Not recorded & $73(8.75)$ & $18997(18.37)$ & 0.45 & $0.35-0.58$ & $<0.001$ \\
\hline & $<3.0$ & $188(22.54)$ & 33817 (32.71) & Base & & \\
\hline
\end{tabular}


Table 2 Descriptive and univariable logistic regression results (Continued)

\begin{tabular}{|c|c|c|c|c|c|c|}
\hline \multirow{5}{*}{$\begin{array}{l}\text { Age category (years) (pre-existing cases } \\
\text { included as not-recorded) }\end{array}$} & $3.0-5.9$ & $136(16.31)$ & $26381(25.51)$ & 0.93 & $0.74-1.16$ & 0.504 \\
\hline & $6.0-8.9$ & $232(27.82)$ & $20062(19.40)$ & 2.08 & $1.71-2.52$ & $<0.001$ \\
\hline & $9.0-11.9$ & $172(20.62)$ & $13307(12.87)$ & 2.33 & $1.89-2.86$ & $<0.001$ \\
\hline & $\geq 12.0$ & $106(12.71)$ & 9633 (9.32) & 1.98 & $1.56-2.51$ & $<0.001$ \\
\hline & Not recorded & $0(0.00)$ & 199 (0.19) & $\sim$ & $\sim$ & $\sim$ \\
\hline \multirow[t]{3}{*}{ Sex } & Female & $382(45.80)$ & $49180(47.68)$ & Base & & \\
\hline & Male & $452(54.20)$ & $53933(52.29)$ & 1.08 & $0.94-1.24$ & 0.276 \\
\hline & Not recorded & $0(0.00)$ & $26(0.03)$ & $\sim$ & $\sim$ & $\sim$ \\
\hline \multirow[t]{3}{*}{ Neuter status } & Entire & $123(14.75)$ & $13875(13.42)$ & Base & & \\
\hline & Neutered & $436(52.28)$ & $46050(44.54)$ & 1.07 & $0.87-1.31$ & 0.521 \\
\hline & Not recorded & 275 (32.97) & $43474(42.04)$ & 0.71 & $0.58-0.88$ & 0.002 \\
\hline \multirow[t]{3}{*}{ Insurance } & Non-insured & 190 (22.78) & $29062(28.11)$ & Base & & \\
\hline & Insured & 331 (39.69) & 31840 (30.79) & 1.59 & $1.33-1.90$ & $<0.001$ \\
\hline & Not recorded & $313(37.53)$ & $42497(41.10)$ & 1.13 & $0.94-1.35$ & 0.197 \\
\hline
\end{tabular}

Descriptive and univariable logistic regression results for risk factors associated with diagnosis of corneal ulcerative disease in dogs attending primary-care veterinary practices in England. Percentages shown in brackets

${ }^{\mathrm{a}} \mathrm{Cl}$ confidence interval

Pain associated with CUD was recorded in the clinical notes of $385(46.2 \%)$ cases. At least one analgesic agent was used in 455 (54.6\%) of dogs. Overall, $576(69.1 \%)$ of CUD cases had either pain recorded in their notes and/ or received an analgesic agent. Of the 455 dogs receiving pain management, the most commonly used analgesic agents were non-steroidal anti-inflammatory drugs $(n=$ $421,92.5 \%)$, cycloplegics $(36,7.9 \%)$, opioids $(25,5.5 \%)$ and local anaesthetic $(24,5.3 \%)$. Analgesia was more likely to be used in brachycephalic types than in nonbrachycephalic types (62.34\% versus $51.43 \%$ respectively, $P=0.004)$. Analgesia usage did not differ between spaniel and non-spaniel types $(P=0.184)$.

Surgical CUD management was used in $142(17.0 \%)$ cases. Surgery was less likely to be performed on spaniel compared with non-spaniel types $(7.69 \%$ versus $18.75 \%$ respectively, $P=0.002$ ). The probability of surgery did not differ between brachycephalic types and nonbrachycephalic types $(P=0.137)$. Of the 834 cases overall, $62(7.4 \%)$ were referred for advanced clinical management, the owners sought a second opinion at another primary-care practice in $13(1.6 \%)$ cases, and $3(0.4 \%)$ were transferred to the charity sector. There was no significant difference in the probability of referral between brachycephalic $(24 / 239,10.04 \%)$ and non-brachycephalic dogs $(38 / 595,6.39 \%)(P=0.069))$, or between spaniels $(9 / 130$, $6.92 \%)$ and non-spaniels $(53 / 704,7.53 \%)(P=0.809)$. Dogs that were referred were more likely to receive surgical management $(26 / 62,41.94 \%)$ than dogs that were not referred $(116 / 772,15.03 \%)(P<0.001)$.

At the end of the study period, the clinical records indicated CUD resolution in 658 (78.9\%) cases. Brachycephaly was not associated with the probability of resolution $(P=0.392)$ but spaniels were less likely to have resolved than non-spaniels $(P=0.004)$. Resolution was less likely in dogs that were referred $(67.7 \%)$ than in those that were not referred $(81.0 \%)(P<0.001)$. Recurrence of CUD in the same eye following resolution during the study period was recorded in $45(5.4 \%)$ of cases. Ninety-four (11.3\%) of the CUD cases died of all causes during the study period, with 74 (78.7\%) of these deaths involving euthanasia. CUD was recorded as contributing to $10(13.5 \%)$ of these 74 euthanasia decisions.

Univariable logistic regression modelling identified five variables that were liberally associated with CUD and were further evaluated in the main multivariable logistic regression modelling: breed, bodyweight relative to breed mean, age, neuter and insurance. The final main multivariable model retained three risk factors: breed, age, and insurance. No biologically significant interactions were identified in the final model. The final model showed good discrimination (area under the ROC curve: 0.7368). After accounting for the effects of the other variables evaluated, 15 breeds showed increased odds of CUD compared with crossbred dogs. The breeds with the highest odds included the Pug (OR: 19.05, 95\% CI 13.45-26.97, $P<0.001$ ), Boxer (OR: $12.12,95 \%$ CI 8.77-16.76, $P<0.001$ ) and Shih Tzu (OR: 10.04, 95\% CI 7.30-13.80, $P<0.001$ ). Compared with dogs aged $<3.0$ years, dogs aged $6.0-8.9$ years had 2.24 times the odds (95\% CI $1.82-2.75, P<0.001)$ of CUD. Insured dogs had 1.6 (95\% CI 1.33-1.92, $P<0.001)$ times the odds of CUD compared with uninsured dogs (Table 3).

As described in the methods, four variables (purebred, spaniel-brachycephalic, Kennel Club breed group and adult bodyweight) individually replaced the breed 
variable in the final multivariable model. Purebred dogs were strongly associated with CUD, showing 2.23 times the odds $(95 \%$ CI $1.84-2.87, P<0.001)$ compared with crossbred dogs. In support of the study hypothesis, brachycephalic type had 11.18 (95\% CI 8.72-14.32, $P<$ $0.001)$ times the odds and spaniel type had 3.12 (95\% CI 2.37-4.10, $P<0.001)$ times the odds compared with crossbred dogs. Four of the seven Kennel Club breed groups showed higher odds of CUD compared with dogs of breeds that are not recognized by the Kennel Club: Utility, Working, Toy and Terrier. The odds of CUD decreased as adult bodyweight increased (Table 4).

\section{Discussion}

This is the first study to explore the wider presentation of CUD in dogs attending primary-care practices by analysing clinical data from a multicentre primary-care research database. Previous studies have mainly relied on referral populations [5-10]. This current study design aimed to explore primary-care clinical data in order to reduce selection bias issues that frequently limit the generalisabilty of research based on referral caseloads [11]. It is also the first study to confirm brachycephalic and spaniel breed types as major risk factors for the diagnosis of CUD in general practice caseloads. This is useful evidence for ophthalmologists to support their advice to referring general practices as well as for primary-care veterinarians tasked with diagnosing and managing dogs with CUD.

The power of research to report precise prevalence estimates and to explore multiple risk factors is dependent on large study sample sizes [47]. The case counts used in previous publications on CUD have generally been small $[5,7-10,16-29]$. Although the overall study population of some of these earlier studies may have included up to 200 animals [5, 21, 26], the count of CUD cases within each population, when clearly presented, often dropped to much lower numbers [5]. Furthermore, many of these earlier studies only included referral caseloads, which introduces inevitable selection bias into the study design [11]. In contrast, the present study analysed data from 104,233 dogs attending primary-care practices, among which there were 834 patients recorded with CUD. This, to the best of the authors' knowledge, makes the present study the largest of its kind, and the only one based on an exclusively primary-care practice population, and therefore offers a CUD clinical perspective that has previously not been reported [12, 48].

The results of the present study show strong evidence through the analysis of large numbers that purebred dogs in the general population are more likely to be diagnosed with CUD than non-purebred dogs (2.23 times the odds), and that brachycephalic (11.18 times the odds) and spaniel types (3.12 times the odds) in particular appear especially predisposed to diagnosis of CUD. Previous studies on smaller, referral populations have also suggested that brachycephalic breeds and those non-brachycephalic breeds that also have prominent eyes could be overrepresented for CUD [7, 9, 31, 49]. Brachycephalic conformation has been associated with reduced numbers of corneal nerve endings [50]. Studies have also shown that corneal sensation as measured by corneal aesthesiometry is reduced in brachycephalic dogs and cats $[50,51]$. It is also known that denervation of the cornea can lead to pathology of the corneal epithelium [52-55], and that corneal limbal stem cell niches require a nurturing relationship with corneal innervation [56]. Therefore, reduced corneal sensation and fewer numbers of corneal nerves that are physiologically associated with the brachycephalic skull conformation may lead to an increase in, or at least predispose to, the development of CUD, and might at least partially explain why brachycephalic breeds were more likely to be diagnosed with CUD in the present study. However, other common periocular features of brachycephalic dogs, such as the presence of a large palpebral aperture and a shallow socket, may additionally play interactive roles in the pathogenesis of CUD by promoting greater exposure of the less sensitive cornea $[31,57,58]$. It is interesting to note that in the current study, spaniels also had an increased risk of CUD. Morphologically, some spaniels have a relatively large interpalpebral fissure and shallow socket when compared to non-spaniel and nonbrachycephalic breeds, and it is possible this conformation might play a role in development of CUD in spaniel breeds too [31]. Because the precise factors that promote CUD in brachycephalic and spaniel breeds remains speculative, these aspects warrant further study and may offer possible breeding selection routes towards reducing the impact of CUD on the welfare of these breed types.

The current study showed that compared with crossbred dogs, the Pug, Boxer and Shih Tzu showed very high breed predilections for CUD. It is notable that these predisposed breeds were all brachycephalic with worryingly high results and therefore a major group effort from ophthalmologists, primary-care veterinarians, researchers, owners and breeders is urgently needed to reduce CUD in these highly-predisposed breeds. A previous study on the overall disorder burden of Pugs reported corneal disorders as the second most common individual condition of Pugs (8.72\% prevalence) and identified ophthalmological disorders as the most common group of disorders in Pugs overall (16.25\% prevalence) [59].

Adverse welfare effects are an important aspect of CUD in dogs from a perspective of presence of pain and mortality [3]. Retention of vision and loss of the eye may 
Table 3 Final multivariable logistic regression results

\begin{tabular}{|c|c|c|c|c|}
\hline Variable & Category & Odds ratio & $95 \% \mathrm{Cl}^{\mathrm{a}}$ & $P$-value \\
\hline \multirow[t]{20}{*}{ Common breeds } & Crossbreed & Base & & \\
\hline & Pug & 19.05 & $13.45-26.97$ & $<0.001$ \\
\hline & Boxer & 12.12 & $8.77-16.76$ & $<0.001$ \\
\hline & Shih Tzu & 10.04 & $7.30-13.80$ & $<0.001$ \\
\hline & Cavalier King Charles Spaniel & 6.10 & $4.36-8.54$ & $<0.001$ \\
\hline & Bulldog & 7.76 & 4.69-12.84 & $<0.001$ \\
\hline & King Charles Spaniel & 5.62 & $2.98-10.62$ & $<0.001$ \\
\hline & Lhasa Apso & 4.97 & $3.01-8.22$ & $<0.001$ \\
\hline & French Bulldog & 7.25 & $3.92-13.42$ & $<0.001$ \\
\hline & West Highland White Terrier & 2.05 & $1.34-3.15$ & 0.001 \\
\hline & Staffordshire Bull Terrier & 2.50 & $1.81-3.45$ & $<0.001$ \\
\hline & Cocker Spaniel & 2.14 & $1.45-3.15$ & $<0.001$ \\
\hline & English Springer Spaniel & 1.93 & $1.17-3.19$ & 0.010 \\
\hline & Border Terrier & 2.00 & $1.06-3.75$ & 0.032 \\
\hline & Chihuahua & 2.54 & $1.50-4.28$ & $<0.001$ \\
\hline & Yorkshire Terrier & 1.78 & $1.13-2.80$ & 0.013 \\
\hline & Jack Russell Terrier & 1.45 & $1.00-2.11$ & 0.050 \\
\hline & Border Collie & 0.96 & $0.53-1.77$ & 0.907 \\
\hline & Labrador Retriever & 0.57 & $0.36-0.90$ & 0.016 \\
\hline & Other purebreds & 1.30 & $1.00-1.69$ & 0.052 \\
\hline \multirow{6}{*}{$\begin{array}{l}\text { Age category (years) (pre-existing } \\
\text { cases included as non-recorded) }\end{array}$} & $<3.0$ & Base & & \\
\hline & $3.0-5.9$ & 0.96 & $0.76-1.21$ & 0.724 \\
\hline & $6.0-8.9$ & 2.24 & $1.82-2.75$ & $<0.001$ \\
\hline & $9.0-11.9$ & 2.78 & $2.23-3.47$ & $<0.001$ \\
\hline & $\geq 12.0$ & 2.74 & $2.13-3.53$ & $<0.001$ \\
\hline & Not recorded & $\sim$ & & \\
\hline \multirow[t]{3}{*}{ Insurance } & Non-insured & Base & & \\
\hline & Insured & 1.6 & $1.33-1.92$ & $<0.001$ \\
\hline & Not recorded & 1.27 & $1.06-1.54$ & 0.012 \\
\hline
\end{tabular}

Final multivariable logistic regression model for risk factors associated with diagnosis of corneal ulcerative disease in dogs attending primary-care veterinary practices in England

${ }^{\mathrm{a}} \mathrm{Cl}$ confidence interval

also be considered as long-term welfare issues for affected individuals although we do not currently have the objective data on which to base definitive conclusions on these judgements [4]. It is important to check central corneal clarity following CUD management as this impacts on long-term vision [60]. A validated corneal clarity scoring system for veterinary cases exists and its use, as well as the recording of the results obtained into the clinical notes, should be encouraged in all cases of CUD [60]. The present study did not aim to carry out an in-depth analysis of primary-care diagnostics and management for CUD. However, it did aim to extract data on basic clinical management that could assist with welfare evaluations and that could be complemented by future more-focused studies that specifically aim to increase our understanding of primary-care CUD management. Keratoconjunctivitis sicca has been associated with the development of CUD, especially in brachycephalics, and it is recommended that dogs with corneal ulcers undergo tear measurement with Schirmer tear test-1 [7]. In the present study, just $23.7 \%$ of CUD cases underwent Schirmer tear testing.

Evaluation of welfare impact for disorders at a population level is important to assist with evidence-based ranking of disorders and strategising the distribution of limited resources for welfare improvement, for example through regulatory or legislative change, genetic advances or breeding schemes [61]. A GISID (generic illness and severity index for dogs) has been proposed that 
Table 4 Multivariable logistic regression results for variables that replaced breed

\begin{tabular}{|c|c|c|c|c|}
\hline Variable & Category & Odds ratio & $95 \% \mathrm{Cl}^{\mathrm{a}}$ & $P$-value \\
\hline \multirow[t]{2}{*}{ Purebred status } & Crossbred & Base & & \\
\hline & Purebred & 2.23 & $1.84-2.87$ & $<0.001$ \\
\hline \multirow[t]{4}{*}{ Spaniel-brachycephalic } & Crossbred & Base & & \\
\hline & Spaniel & 3.13 & $2.38-4.12$ & $<0.001$ \\
\hline & Brachycephalic & 11.18 & $8.72-14.32$ & $<0.001$ \\
\hline & Purebred other & 1.42 & $1.12-1.79$ & 0.003 \\
\hline \multirow[t]{8}{*}{ Kennel Club Breed Groups } & Breed not Kennel Club recognised & Base & & \\
\hline & Toy & 3.52 & $2.82-4.39$ & $<0.001$ \\
\hline & Utility & 3.99 & $3.16-5.03$ & $<0.001$ \\
\hline & Terrier & 1.83 & $1.44-2.35$ & $<0.001$ \\
\hline & Gundog & 0.97 & $0.75-1.25$ & 0.811 \\
\hline & Hound & 1.04 & $0.66-1.64$ & 0.860 \\
\hline & Pastoral & 0.83 & $0.56-1.22$ & 0.343 \\
\hline & Working & 3.64 & $2.76-4.79$ & $<0.001$ \\
\hline \multirow[t]{6}{*}{ Adult (> 9 months) bodyweight (kg) } & $<10.0$ & 1.88 & $1.47-2.41$ & $<0.001$ \\
\hline & 10.0-19.9 & 1.70 & $1.33-2.18$ & $<0.001$ \\
\hline & 20.0-29.9 & 1.26 & $0.96-1.64$ & 0.090 \\
\hline & $30.0-39.9$ & Base & & \\
\hline & $\geq 40.0$ & 0.69 & $0.45-1.05$ & 0.086 \\
\hline & Not available & 0.73 & $0.53-1.02$ & 0.064 \\
\hline
\end{tabular}

Results for variables that replaced the breed variable in the final multivariable logistic regression model (with age category and insurance status) to evaluate risk factors associated with a diagnosis of corneal ulcerative disease in dogs attending primary-care veterinary practices in England

${ }^{\mathrm{a}} \mathrm{Cl}$ confidence interval

aims to objectively generate welfare risk scores using data on prevalence, duration and severity [61]. The current study contributes some information that may assist efforts to evaluate and rank CUD within the overall spectrum of disorders in dogs. Overall, $69.1 \%$ of CUD cases had either pain recorded in their notes and/or received pain management, suggesting that the attending veterinary surgeons perceived CUD to be a painful condition in a high proportion of cases. The use of surgical management in $17.0 \%$ of the CUD cases in the current study may have also introduced an element of surgical pain for these cases but successful surgery may also have hastened recovery and therefore reduced the overall pain experienced for many dogs [62]. The current study also recorded a recurrence rate of $5.4 \%$ although the followup time was limited and a longer study may lead to increases in this result. CUD also contributed the decision-making for 10 of the 74 (13.5\%) dogs that were euthanased during the study. However, interpretation of ocular pain findings must be taken with great care. Despite the existence of studies on the validation of systemic pain scores for use in companion animals [63-65], there are no scoring systems validated for ocular pain in veterinary species and ocular pain appreciation remains subjective. It is clear, that there is a great need for veterinary professionals to develop a reliable tool to assess ocular pain, which is currently lacking. However, it should be noted that the current study was primarily an epidemiological investigation and therefore cannot fully address the impacts of CUD on the affective state of CUD cases. This would require a multifaceted focus on physiological, behavioural, cognitive and subjective components [66]. Only 62 (7.4\%) cases were referred for CUD management, which means the majority of cases were entirely under primary-care clinical management. Referral, for the most part, aims to offer ophthalmic patients and their owners the assessment and opinion of a dedicated specialist and access to on-site microsurgical facilities for corneal reconstruction if deemed necessary [67]. Despite this, it is worth noting that $81.0 \%$ of the CUD cases that were managed entirely within the primary-care setting had resolved by the end of the study. As mentioned earlier, however, assessment of treatment success of CUD should also include the corneal clarity score achieved after treatment [60]. Although non-referral might appear unrelated to a positive outcome in this study, saving an eye alone does not necessarily equate to treatment success if vision is lost as a result of CUD.

Lastly, it was noted that insured dogs in the current study had 1.6 times the odds of CUD diagnosis compared with non-insured dogs. This tendency towards 
enhanced diagnostic probability in insured animals has now been demonstrated across a wide range of disorders and there is a trend for the diagnostic impact from pet insurance to increase as disorders require more expensive or complicated diagnostic protocols [36, 68-71]. Pet insurance may reduce financial constraints for both the owner and the veterinarian and consequently encourage earlier and more frequent veterinary visits and allow greater diagnostic freedom with consequential gains to animal welfare [72]. It is also possible that owners who purchase pet insurance might have stronger emotional bonds with their animal or higher commitment to providing the best medical therapy regardless of any financial constraints [73].

The study had some limitations. As previously described, these data were not recorded primarily for research purposes and thus were limited by some missing data as well as reliance on accurate and thorough record-keeping of the clinicians [69, 71, 74]. The study included all cases diagnosed with CUD and did not attempt to categorize these based on etiological subsets. The current study defined specific lists of breeds that were described as 'brachycephalic' and 'spaniel' types and aimed for an overall exploration of associations between these types of dogs with CUD. Explanations for the specific selections are included in the methods section but reasoned arguments could equally be made to extend these lists to include other breeds or for the removal of certain breeds from the current lists. The authors accept that changing the breed listings may impact somewhat on the specific results but is unlikely to substantially change the overall inference. Brachycephaly is not a fixed or binary conformational attribute but exists on a continuum from extreme to moderate brachycephalism that can be scored more precisely by newer cephalic index systems derived from various skull width to skull length ratios [44] Additionally, it is worth noting that, not all individuals may necessarily even be brachycephalic among breeds that are traditionally considered as a typically brachycephalic type. Inference was drawn on the opinion of the attending practitioners on the painfulness of CUD by extracting data on whether 'pain' was recorded in the clinical notes and whether analgesia was administered. However, neither of these actions are necessarily proof of true pain and, conversely, lack of recording of pain or failure to use analgesics do not necessarily preclude the presence of pain.

\section{Conclusions}

This is the first study to explore the prevalence and odds of CUD based on skull conformation and breed in a large general population of dogs attending primary-care practice. The results provide evidence for the first time of a strong predisposition to CUD in brachycephalic and spaniel breed types in the general population. The results and conclusions presented here can assist ophthalmologists, general practitioners, breeders and owners by improving breed-based advice about CUD diagnosis, management and prevention.

\section{Abbreviations \\ Cl: Confidence intervals; CUD: Corneal ulcerative disease; EPR: Electronic patient record; KCS: Keratoconjunctivitis sicca; OR: Odds ratio; STT-1: Schirmer tear test-1}

\section{Acknowledgements}

Thanks to Noel Kennedy (RVC) for VetCompass software and programming development. We acknowledge the Medivet Veterinary Partnership Vets4Pets/Companion Care, Blythwood Vets, Vets Now and the other UK practices who collaborate in VetCompass. We are grateful to The Kennel Club, The Kennel Club Charitable Trust and Dogs Trust for supporting VetCompass.

\section{Funding}

DON is supported at the RVC by an award from the Kennel Club Charitable Trust. Neither the Kennel Club Charitable Trust nor the Kennel Club had any input in the design of the study, the collection, analysis and interpretation of data or in writing the manuscript should be declared.

\section{Availability of data and materials \\ The datasets generated and analysed during the current study are not publicly available due to their use in ongoing primary research but subsections may be made available from the corresponding author on reasonable request.}

\section{Authors' contributions}

All authors made substantial contributions to conception and design, acquisition and extraction of data, and to analysis and interpretation of the results. All authors were involved in drafting and revising the manuscript and gave final approval of the version to be published. Each author agrees to be accountable for all aspects of the accuracy or integrity of the work.

\section{Competing interests}

DON is supported at the RVC by an award from the Kennel Club Charitable Trust. The remaining authors have no conflicts of interest to declare.

\section{Consent for publication}

Not applicable.

Ethics approval and consent to participate

Ethical approval was granted by the RVC Ethics and Welfare Committee (reference number 2015/T94).

\section{Publisher's Note}

Springer Nature remains neutral with regard to jurisdictional claims in published maps and institutional affiliations.

\section{Author details}

${ }^{1}$ Pathobiology and Population Sciences, The Royal Veterinary College, Hawkshead Lane, North Mymms, Hatfield, Herts AL9 7TA, UK. ${ }^{2}$ The Royal Veterinary College, Hawkshead Lane, North Mymms, Hatfield, Herts AL9 7TA, UK. ${ }^{3}$ Clinical Science and Services, The Royal Veterinary College, Hawkshead Lane, North Mymms, Hatfield, Herts AL9 7TA, UK. ${ }^{4}$ Speciaslistische

Dierenkliniek Utrecht, Middenwetering 19, Utrecht, Netherlands.

Received: 27 March 2017 Accepted: 8 May 2017

Published online: 15 June 2017

\section{References}

1. Gum GG, MacKay EO. Physiology of the Eye. In: Gelatt KN, Gilger BC, Kern TJ, editors. Veterinary ophthalmology, vol. 1. 5th ed. Oxford: Wiley-Blackwell; 2013. p. 171-207. 
2. Ollivier FJ, Gilger BC, Barrie KP, Kallberg ME, Plummer CE, O'Reilly S, et al. Proteinases of the cornea and preocular tear film. Vet Ophthalmol. 2007 10(4):199-206

3. Ledbetter EC, Gilger BC. Diseases and surgery of the canine cornea and sclera. In: Gelatt KN, Gilger BC, Kern TJ, editors. Veterinary ophthalmology, vol. 2. 5th ed. Oxford: Wiley-Blackwell; 2013. p. 976-1049.

4. Rooney NJ, Sargan DR. Welfare concerns associated with pedigree dog breeding in the UK. Anim Welfare. 2010;19:133-40.

5. Stanley RG, Hardman C, Johnson BW. Results of grid keratotomy, superficial keratectomy and debridement for the management of persistent corneal erosions in 92 dogs. Vet Ophthalmol. 1998;1(4):233-8.

6. Murphy CJ, Marfurt CF, McDermott A, Bentley E, Abrams GA, Reid TW, et al. Spontaneous chronic corneal epithelial defects (SCCED) in dogs: clinical features, innervation, and effect of topical SP, with or without IGF-1. Investig Ophthalmol Vis Sci. 2001:42(10):2252-61.

7. Sanchez RF, Innocent G, Mould J, Billson FM. Canine keratoconjunctivitis sicca: disease trends in a review of 229 cases. J Small Anim Pract. 2007:48(4):211-7.

8. Krecny M, Tichy A, Rushton J, Nell B. A retrospective survey of ocular abnormalities in pugs: 130 cases. J Small Anim Pract. 2015;56(2):96-102.

9. Dorbandt DM, Moore PA, Myrna KE. Outcome of conjunctival flap repair for corneal defects with and without an acellular submucosa implant in 73 canine eyes. Vet Ophthalmol. 2015;18(2):116-22.

10. Dawson C, Naranjo C, Sanchez-Maldonado B, Fricker GV, Linn-Pearl RN, Escanilla $\mathrm{N}$, et al. Immediate effects of diamond burr debridement in patients with spontaneous chronic corneal epithelial defects, light and electron microscopic evaluation. Vet Ophthalmol. 2015;1-5.

11. Bartlett PC, Van Buren JW, Neterer M, Zhou C. Disease surveillance and referral bias in the veterinary medical database. Prev Vet Med. 2010;94(3-4):264-71.

12. McGreevy PD, Nicholas FW. Some practical solutions to welfare problems in dog breeding. Anim Welfare. 1999:8:329-41.

13. Bateson P. Independent inquiry into dog breeding. Cambridge: University of Cambridge; 2010 .

14. Collins LM, Asher L, Summers J, McGreevy P. Getting priorities straight: risk assessment and decision-making in the improvement of inherited disorders in pedigree dogs. Vet J. 2011;189(2):147-54.

15. Sanchez RF. Chapter 12: the cornea. In: Gould D, McLellan GJ, editors. BSAVA manual of canine and feline ophthalmology. 3rd ed. London: BSAVA; 2014.

16. Bentley E, Abrams GA, Covitz D, Cook CS, Fischer CA, Hacker D, et al. Morphology and immunohistochemistry of spontaneous chronic corneal epithelial defects (scced) in dogs. Investig Ophthalmol Vis Sci. 2001;42(10): 2262-9

17. Gervais KJ, Pirie CG, Ledbetter EC, Pizzirani S. Acute primary canine herpesvirus-1 dendritic ulcerative keratitis in an adult dog. Vet Ophthalmol. 2012;15(2):133-8.

18. Van Der Woerdt A. Adnexal surgery in dogs and cats. Vet Ophthalmol. 2004; 7(5):284-90.

19. Read RA, Broun HC. Entropion correction in dogs and cats using a combination Hotz-Celsus and lateral eyelid wedge resection: results in 311 eyes. Vet Ophthalmol. 2007;10(1):6-11.

20. D'Anna N, Sapienza JS, Guandalini A, Guerriero A. Use of a dermal biopsy punch for removal of ectopic cilia in dogs: 19 cases. Vet Ophthalmol. 2007; 10(1):65-7.

21. Sansom J. Keratoconjunctivitis sicca in the dog: a review of two hundred cases. J Small Anim Pract. 1985;26(3):121-31.

22. Sansom J, Blunden T. Calcareous degeneration of the canine cornea. Vet Ophthalmol. 2010;13(4):238-43.

23. Christmas R. Management of chemical burns of the canine cornea. Can Vet J. 1991;32(10):608-12.

24. Vanore M, Chahory S, Payen G, Clerc B. Surgical repair of deep melting ulcers with porcine small intestinal submucosa (SIS) graft in dogs and cats. Vet Ophthalmol. 2007;10(2):93-9.

25. Paulsen ME, Kass PH. Traumatic corneal laceration with associated lens capsule disruption: a retrospective study of 77 clinical cases from 1999 to 2009. Vet Ophthalmol. 2012;15(6):355-68.

26. Dawson C, Sanchez RF. A prospective study of the prevalence of corneal surface disease in dogs receiving prophylactic topical lubrication under general anesthesia. Vet Ophthalmol. 2016;19(2):124-9.

27. Spivack RE, Elkins AD, Moore GE, Lantz GC. Postoperative complications following TECA-LBO in the Dog and Cat. J Am Anim Hosp Assoc. 2013;49(3):160-8.

28. Attali-Soussay $\mathrm{K}$, Jegou J-P, Clerc B. Retrobulbar tumors in dogs and cats: 25 cases. Vet Ophthalmol. 2001;4(1):19-27.
29. van der Woerdt A. Orbital inflammatory disease and pseudotumor in dogs and cats. Vet Clin N Am Small Anim Pract. 2008;38(2):389-401.

30. Gough A, Thomas A. Breed predispositions to disease in dogs and cats. 2nd ed. Chicester: Wiley-Blackwell; 2010.

31. Packer RMA, Hendricks A, Burn CC. Impact of facial conformation on canine health: corneal ulceration. PLOS ONE. 2015;10(5):1-16.

32. Tolar EL, Hendrix DVH, Rohrbach BW, Plummer CE, Brooks DE, Gelatt KN. Evaluation of clinical characteristics and bacterial isolates in dogs with bacterial keratitis: 97 cases (1993-2003). J Am Vet Med Assoc. 2006;228(1):80-5.

33. Tetas Pont R, Matas Riera M, Newton R, Donaldson D. Corneal and anterior segment foreign body trauma in dogs: a review of 218 cases. Vet Ophthalmol. 2015;19(5):386-97.

34. O'Neill DG, Church DB, McGreevy PD, Thomson PC, Brodbelt DC. Prevalence of disorders recorded in dogs attending primary-care veterinary practices in England. PLOS ONE. 2014;9(3):1-16.

35. The VeNom Coding Group. VeNom Veterinary Nomenclature [http://www. venomcoding.org]. Accessed 12 May 2017.

36. O'Neill DG, Scudder C, Faire JM, Church DB, McGreevy PD, Thomson PC, et al. Epidemiology of hyperadrenocorticism among 210,824 dogs attending primary-care veterinary practices in the UK from 2009 to 2014. J Small Anim Pract. 2016:57(7):365-73.

37. Pearce N. Classification of epidemiological study designs. Int J Epidemiol. 2012:41(2):393-7.

38. Epi Info 7 CDC. Centers for Disease Control and Prevention (US): Introducing Epi Info 7 [http://wwwn.cdc.gov/epiinfo/7]. Accessed 12 May 2017

39. Irion DN, Schaffer AL, Famula TR, Eggleston ML, Hughes SS, Pedersen NC. Analysis of genetic variation in 28 dog breed populations with 100 microsatellite markers. J Hered. 2003;94(1):81-7.

40. Scott M, Flaherty D, Currall J. Statistics: how many? J Small Anim Pract. 2012 53(7):372-6.

41. Packer RMA, Hendricks A, Tivers MS, Burn CC. Impact of facial conformation on canine health: brachycephalic obstructive airway syndrome. PLoS ONE. 2015:10(10):e0137496.

42. Crane C, Rozanski EA, Abelson AL, de Laforcade A. Severe brachycephalic obstructive airway syndrome is associated with hypercoagulability in dogs. J Vet Diagn Investig. 2017;1040638717703434. doi: https://doi.org/10.1177/ 1040638717703434.

43. Caccamo R, Buracco P, La Rosa G, Cantatore M, Romussi S. Glottic and skull indices in canine brachycephalic airway obstructive syndrome. BMC Vet Res. 2014;10(1):12.

44. Georgevsky D, Carrasco JJ, Valenzuela M, McGreevy PD. Domestic dog skull diversity across breeds, breed groupings, and genetic clusters. J Vet Behav Clin Appl Res. 2014;9(5):228-34.

45. The Kennel Club. Breed Information Centre [http://www.thekennelclub.org uk/services/public/breed/]. Accessed 12 May 2017

46. Kirkwood BR, Sterne JAC. Essential medical statistics. 2nd ed. Oxford: Blackwell Science; 2003.

47. Dohoo I, Martin W, Stryhn H. Veterinary epidemiologic research. 2nd ed. Charlottetown: VER Inc; 2009

48. O'Neill D, Church D, McGreevy P, Thomson P, Brodbelt D. Approaches to canine health surveillance. Canine Genet Epidemiol. 2014;1 (1):2.

49. Lacerda RP, Peña Gimenez MT, Laguna F, Costa D, Ríos J, Leiva M. Corneal grafting for the treatment of full-thickness corneal defects in dogs: a review of 50 cases. Vet Ophthalmol. 2016:1-10.

50. Barrett $P$, Scagliotti $R$, Merideth $R$, Jackson P, Alarcon F. Absolute corneal sensitivity and corneal trigeminal nerve anatomy in normal dogs. Prog Vet Comp Ophthalmol. 1991;1(4):245-54.

51. Blocker T, Van Der Woerdt A. A comparison of corneal sensitivity between brachycephalic and Domestic Short-haired cats. Vet Ophthalmol. 2001;4(2):127-30.

52. Cavanagh HD, Colley AM. The molecular basis of neurotrophic keratitis. Acta Ophthalmol. 1989;67(S192):115-34

53. Touhami A, Grueterich M, Tseng SCG. The role of ngf signaling in human limbal epithelium expanded by amniotic membrane culture. Investig Ophthalmol Vis Sci. 2002;43(4):987-94.

54. Yamada N, Yanai $R$, Inui M, Nishida T. Sensitizing effect of Substance $P$ on corneal epithelial migration induced by IGF-1, fibronectin, or interleukin-6. Investig Ophthalmol Vis Sci. 2005:46(3):833-9.

55. Ueno H, Ferrari G, Hattori T, Saban DR, Katikireddy KR, Chauhan SK, et al. Dependence of corneal stem/progenitor cells on ocular surface innervation. Investig Ophthalmol Vis Sci. 2012;53(2):867-72 
56. Luesma MJ, Gherghiceanu M, Popescu LM. Telocytes and stem cells in limbus and uvea of mouse eye. J Cell Mol Med. 2013;17(8):1016-24.

57. McGreevy P, Grassi TD, Harman AM. A strong correlation exists between the distribution of retinal ganglion cells and nose length in the dog. Brain Behav Evol. 2004;63(1):13-22.

58. Maggs DJ, Miller PE, Ofri R, Slatter DHFovo. Slatter's fundamentals of veterinary ophthalmology. 4th ed. Philadelphia; London: Elsevier Saunders; 2008.

59. O'Neill DG, Darwent EC, Church DB, Brodbelt DC. Demography and health of Pugs under primary veterinary care in England. Canine Genet Epidemiol. 2016:3(1):1-12.

60. Sanchez RF, Dawson C, Matas Riera M, Escanilla N. Preliminary results of a prospective study of inter-and intra-user variability of the Royal Veterinary College corneal clarity score (RVC-CCS) for use in veterinary practice. Vet Ophthalmol. 2016;19(4):313-8.

61. Collins LM, Asher L, Summers JF, Diesel G, McGreevy PD. Welfare epidemiology as a tool to assess the welfare impact of inherited defects on the pedigree dog population. Anim Welfare. 2010;19:67-75.

62. Hansen BD. Assessment of pain in dogs: veterinary clinical studies. ILAR J. 2003:44(3):197-205.

63. Holton LL, Scott EM, Nolan AM, Reid J, Welsh E, Flaherty D. Comparison of three methods used for assessment of pain in dogs. J Am Vet Med Assoc. 1998:212(1):61-6.

64. Firth AM, Haldane SL. Development of a scale to evaluate postoperative pain in dogs. J Am Vet Med Assoc. 1999;214(5):651-9.

65. Holton L, Reid J, Scott EM, Pawson P, Nolan A. Development of a behaviour-based scale to measure acute pain in dogs. The Veterinary Record. 2001;148(17):525-31.

66. Paul ES, Harding EJ, Mendl M. Measuring emotional processes in animals: the utility of a cognitive approach. Neurosci Biobehav Rev. 2005;29(3):469-91.

67. Gelatt KN. Veterinary ophthalmology: our past, present and future. Bulletin de l'Academie Veterinaire de France. 2008;161(4):299-306.

68. Taylor-Brown FE, Meeson RL, Brodbelt DC, Church DB, McGreevy PD, Thomson PC, et al. Epidemiology of cranial cruciate ligament disease diagnosis in dogs attending primary-care veterinary practices in England. Vet Surg. 2015;44(6):777-83.

69. Shoop S, Marlow S, Church D, English K, McGreevy P, Stell A, et al. Prevalence and risk factors for mast cell tumours in dogs in England. Canine Genet Epidemiol. 2015;2(1):1.

70. O'Neill DG, Elliott J, Church DB, McGreevy PD, Thomson PC, Brodbelt DC. Chronic kidney disease in dogs in UK veterinary practices: prevalence, risk factors, and survival. J Vet Intern Med. 2013;27(4):814-21.

71. O'Neill DG, Meeson RL, Sheridan A, Church DB, Brodbelt DC. The epidemiology of patellar luxation in dogs attending primary-care veterinary practices in England. Canine Genet Epidemiol. 2016;3(1):1-12

72. Egenvall A, Nødtvedt A, Penell J, Gunnarsson L, Bonnett BN. Insurance data for research in companion animals: benefits and limitations. Acta Vet Scand. 2009;51:42.

73. Shore ER, Douglas DK, Riley ML. What's in it for the companion animal? Pet attachment and college students' behaviors toward pets. J Appl Anim Welf Sci. 2005:8(1):1-11.

74. Mattin MJ, Boswood A, Church DB, López-Alvarez J, McGreevy PD, O’Neill $D G$, et al. Prevalence of and risk factors for degenerative mitral valve disease in dogs attending primary-care veterinary practices in England. J Vet Intern Med. 2015;29(3):847-54.

\section{Submit your next manuscript to BioMed Central and we will help you at every step:}

- We accept pre-submission inquiries

- Our selector tool helps you to find the most relevant journal

- We provide round the clock customer support

- Convenient online submission

- Thorough peer review

- Inclusion in PubMed and all major indexing services

- Maximum visibility for your research

Submit your manuscript at www.biomedcentral.com/submit

) Biomed Central 